\title{
Maturity Model for Cognitive Computing Systems in the Public Sector
}

\author{
Kevin C. Desouza \\ School of Management \\ QUT Business School \\ Queensland University of \\ Technology \\ $\underline{\text { kevin.desouza@qut.edu.au }}$
}

\author{
Franziska Götz \\ Chair of International Management \\ University of Bayreuth \\ franziska.goetz@uni-bayreuth.de \\ Centre for Future Enterprise \\ QUT Business School, Queensland \\ University of Technology \\ franziska.goetz@qut.edu.au
}

\author{
Gregory S. Dawson \\ School of Accountancy \\ W. P. Carey School of Business \\ Arizona State University \\ gregorySDawson@gmail.com
}

\begin{abstract}
Thanks to their enormous potential for creating more efficient processes and solving vexing problem, cognitive computing systems (CCSs) are increasingly prevalent in the public sector. However, their full deployment is stymied by all of the problems faced by private firms (e.g. organizational issues, people issues and technology issues) as well as problems that are unique to the public sector including stakeholder groups with conflicting goals and a demand for full transparency. In this study, we develop a publicsector centric maturity model approach to CCSs that acknowledges and addresses these problems while providing a path to evaluate, assess and guide CCS initiatives. By following this model, the public sector can reap the rewards of CCS deployment and provide better outcomes for its citizenry.
\end{abstract}

\section{Introduction}

According to a recent IBM survey, $87 \%$ of government executives agreed that "cognitive computing plays a disruptive role in their organizations, and that they intend to invest in cognitive capabilities" [35]. CCSs incorporate discoveries across the fields of cognitive science, artificial intelligence, computer and information science [42] and include an array of technologies including Artificial Intelligence (AI), Big Data Analytics (BDA), Data Visualization (DV), Deep Learning (DL), Machine Learning (ML), and Natural Language Processing (NLP) [24]. At present, public agencies around the world are increasingly investing significant resources into CCSs [37].

CCSs offer several key value propositions for public agencies. CCSs can 1) increase the efficiency of administrative processes through automation, 2) enhance the design, development, and evaluation of public policies by leveraging big data to generate novel insights, 3) increase the quality of citizen engagement and the delivery of public services through digital agents such as chatbots, and 4) reshape the nature of public workforce and design of public agencies to one in which innovation is prioritized.

CCSs projects in the public sector present unique challenges [17]. First, decisions must be made transparently, as they are paid for with taxpayer money. Second, stakeholder groups differ more widely, which leads to different conflicts of interest. Third, not only do AI projects in the public sector have to improve the public good, they also must create public value. Fourth, the public sector must cope with complex economic, legal, political, and social elements. Finally, the organization's strategy as well as human resources may also be in conflict with the implementation of AI technologies and therefore cause organizational and management challenges.

While investments in CCSs continue to rise in the public sector, we are yet to see significant public value from these systems. A possible reason for this could be the lack of required maturity CCSs initiatives. Consider two examples. First, an AI-based algorithm, which determines whether an offender was likely to reoffend, and the results of this was used to establish longer prison sentences for those that the model said had a higher risk to reoffend. While the algorithm may have been factually correct, it had the net impact of disproportionally sentencing minorities to longer prison sentences than whites; clearly an example of inappropriate racial profiling [27]. Second, in San Diego, the city's law enforcement has collected more than 65,000 facial scans to match them against a database of mug shots. So far, the outcome of this undertaking is unclear, as neither a criminal prosecution nor a single arrest has resulted from the program [31]. These immature efforts are costly to taxpayers and are not isolated incidents.

In short, there is a need to develop models in order to understand CCSs critical components and to provide guidance to agency managers about their 
current CCSs and how to mature them. Maturity models, such as the one we develop in this paper, can serve as instruments to compare, evaluate and benchmark the further development and success of the implementation of digital measures in public sector institutions [2]. Given the robust differences between the private and public sector, it is simply not possible to port findings from the private sector into the public sector and thus there is a need to develop a public sector centric-model. As such, this maturity model should allow CCSs to contribute positively in the public sector.

\section{Background}

\section{Maturity models}

While it is unclear as to who first developed the notion of a maturity model, most researchers credit Crosby [12], who outlined a five-stage quality management grid (QMG), as having done so. The model developed in this study is an evolutionary model, which gradually increases in complexity and perfection over time in a process called amplification [40]. Perhaps the most well-known maturity model is the Software Engineering Institute's (SEI) Capability Maturity Model (CMM). [25]. The CMM is a five-level improvement model.

Maturity models represent a way to measure the capabilities of an organization and outline the logical and desired evolutionary paths towards maturity. The levels are arranged in a way that capacity at the lower levels forms a strong foundation on which an organization can move to the upper levels [25]. The various levels represent initial, repeatable, defined, managed, and optimizing phases of software capability. Various frameworks have been proposed for the development of maturity models. As an example, consider Bedeley et al [7] who outlines the following guidelines. First, the process of developing a maturity model is iterative, as solutions in the process are proposed, evaluated, refined and improved iteratively. This procedure also applies to all principles and premises associated with the development of the maturity model as well as the quality and effectiveness of the artefacts to be evaluated. Second, the harmonization and application of different research methods must be considered. Third, the model needs to demonstrate relevance to the problem and to the domain and, as a result, continues its iteration. Through this process, due to its iterative character, transitions occur, which needs to be incorporated in the model as well.

\section{Methodology}

We employed a design science approach as outlined by Peffers et al [32] (Fig. 1). Given the state of CCSs deployments in the public sector, we contacted practitioners for insights on how to increase their effectiveness and efficiency. Hence, our research entry point was problem-centered initiation [32].

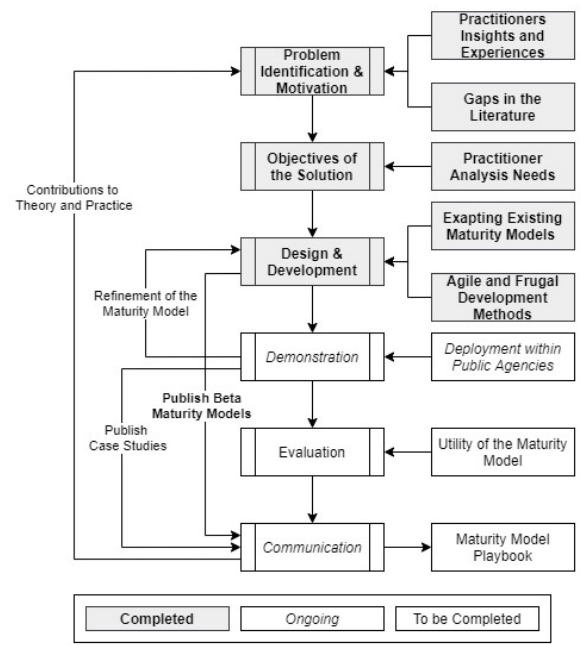

Figure 1-Research process

Problem identification and motivation was conducted by collating insights from practitioners on the need for a maturity model. Specifically, as part of a previous research project [15] that examined challenges and opportunities with CCSs deployments in the public sector, we spoke with over 25 senior IT executives in the public sector. Insights collected from these interviews heightened the need for a maturity framework. In addition, a comprehensive search of the literature was conducted to find existing maturity models on a) CCSs, b) digital transformation, and c) egovernment. Our analysis revealed that there were no existing models that examined the deployment of CCSs in the publics sector and, given the need for domain specific research models [6], we started the development of one within this domain.

Objectives of the solution were identified through conducted 12 new interviews with senior IT executives. These interviews focused on a) the key domains that need to be covered when examining maturity of CCSs, b) the levels of maturity, and c) key enablers and barriers to CCSs maturity.

Design and Development occurred in an iterative manner. We adhered to the principles of agile and frugal development. Specifically, agility was adhered to by creating prototypical maturity models and collecting feedback from practitioners every three weeks. Frugality ensured that we were efficient in 
utilizing resources (e.g. design time, feedback on prototypes, etc.). We also engaged in exaptation [23], which is the drawing on existing maturity models in other domains to create maturity levels. In all, we conducted four design and development cycles and involved soliciting feedback from over 20 seasoned public managers. During each cycle, components of the domains to evaluate CCSs maturity and the progression across the maturity levels were refined. One example of such a refinement is the completion of the second cycle: the model had five domains (big data, computational systems, analytical capacity, innovation climate, and strategic visioning) while the final one has six including governance and ethical frameworks. Within domains, there was refinement of which key elements were captured and how to increase their precision and progression across the levels. All aspects of the model were subjected to this process.

We are currently in the demonstration phase where the model is being used to assess CCS maturity in four public agencies. Guidelines on how to use the maturity model have been drafted and are being deployed within agencies. We intend to draft several case studies to demonstrate how the maturity model was used and what the outcomes could be. Evaluation will commence once we are satisfied with the efficacy of the usage guidelines and we intend to use a new set of experts to do so. Our intention is to evaluate the utility of both the maturity model and the usage guidelines in the future using several cases from the public sector. Communication of our outcomes is ongoing. This conference paper is an example of one output as part of this activity. We have already presented our maturity models at four international forums that brought together public sector professionals who have an interest in digital transformation. We are currently completing a report that will outline the maturity framework and the usage guidelines for practitioners in the public sphere.

\section{CCSs Maturity Model}

The maturity model has two dimensions (see Table 1). The horizontal dimension comprises all elements of CCS that are to be critically assessed. The vertical dimension outlines the different maturity levels. The elements to be assessed can in turn be divided into two domains: technical and organizational. The technical domain comprises big data, computational systems, and analytical capacity. The organizational domain includes innovation climate, governance and ethical frameworks, and strategic visioning.

\subsection{Technical Domain}

Big data

Due to the enormous amount of data being produced on a daily basis, human-analysis is not only time-consuming, but also inefficient [24]. The term 'big data' describes this enormous amount of data [20] and includes the storage of different data types such as image, text and video [28]. CCSs are therefore helpful tools to provide autonomous data collecting, screening and analyzing [24], also known as the term 'big data analytics" [10]. Big Data is "substantial for egovernment services" [8], can stimulate collaboration, generate real-time solutions and boost greater openness [8]. When leveraging data in the public sector, we found several issues. First, data is often captured in forms that are not easily analyzable by machine [18]. Second, data is often locked in different agencies and even within agencies data can be held in various departments and are not easily integrated [38]. Third, is the way we think about the value of data. While governments have done much in the space of open data and even promoting hackathons that engage the public in analyzing data [41], within agencies there is still a challenge to build robust data sets that have the necessary scale for CCSs [18]. Fourth, to tackle complex social issues (e.g. trafficking of individuals, recidivism, and homelessness), we need data that may reside across multiple agencies in the public sector [18]. Here the challenge exists as to who does the integration and who holds the data (agencies refuse to share their data as they view it as a loss of power) [41]. Together, the data are the raw materials for the CCS.

\section{Computational systems}

Computational systems are the engines for CCSs as they process inputs and generate outputs. In this case, computational systems run machine-learning algorithms and build semi- and fully-autonomous systems. In the public sector, there are several issues one has to contend with when it comes to computational systems. First, a large part of the IT budget is spent on keeping current systems running [1]. Second, there is a persistence issue in the use of legacy systems [29]. Dawson et al. [1] mention in their study on IT Modernization in Government different examples on how to improve legacy systems in the public sector. Third, governments have only recently unlocked the use of cloud computing, as they still prefer to own rather than rent computational systems [15]. Fourth, the acquisition of new technologies is still complex and cumbersome [13]. Fifth, the security of these computer systems is a key issue when it comes to opening access to them. Allowing more people and third parties to have system access can entail considerable security risks [39]. 


\section{Analytical capacity}

This is the human element when it comes to designing, developing, and deploying CCSs [30]. Talent is needed, which is in scare supply [14]. In addition, public agencies cannot compete with remuneration within the private sector [14]. But other factors also play an important role. First, while governments have dedicated R\&D Labs in various departments (most notably in the Defense, e.g. US Army Research Labs, Office of Naval Research), these units work on specific technologies that can help the front lines for the most part (e.g. building a better weapon or mining data to discover terrorist) [3]. They do not work on CCSs issues that are needed for the socalled back office functions. Therefore, the analytical capacity is concentrated in these areas but the vast majority of agencies do not see the benefit [11]. Second, when public agencies work with external partners, they do so in mostly episodic fashion. So, they run a hackathon [41] or a crowdsourcing competition such as the "Idea Factory" of the Transportation Security Administration (TSA) in the USA or the Victoria government's VPS Hub [5]. However, these efforts are one-off and never fully get integrated into the analytical capacity of the agency [28]. Third, due to budget cuts, public agencies have limited ability to invest in new talent [37].

\subsection{Organizational Domain}

\section{Innovation climate}

Innovation is a key driver to improve efficiency and performance in the public sector [33]. Thus, public sector agencies need to have the capacity to stimulate innovation and progress especially when it comes to technology-driven innovation. All the factors mentioned above, for example, the data challenges and the legacy computational systems impact the ability of these agencies to innovate [33]. Despite all these challenges, innovation is still possible [16]. But, innovation with technology requires an appetite to promote experimentation [33] and even have the capacity to deploy technologies in controlled environments to learn from their deployment [22]. Moreover, innovation initiatives are contingent upon citizens 'willingness to adopt this innovation [23]. Agencies need ways to involve external stakeholder (e.g. citizens, businesses) into the innovation process [26]. Again there are regulatory and administrative challenges to open up innovation [4].

\section{Governance and Ethical Frameworks}

E-Government fosters accessibility of public services, efficiency, improved services and transparency [21]. Ethical frameworks ensure in general that individual rights are respected [21]. In this context, ethics on e-Governance aim to secure two main areas: the reflection of security as well as the insurance of privacy. Because AI and ICT not only facilitate and enable access, but also the collection of data, there is a particular need for protection when CCSs are used in the public sector, which exchanges data and information of different types, to different groups and of different relevance [9]. Hence, the "egovernance requires a considerable increase in regulation and policy making abilities" [21]

\section{Strategic visioning}

The last concept looks at how CCSs are incorporated into the long-term thinking of agencies [19]. Strategic visioning and plans are thus key artefacts in the public sector [34]. Sousa et al. state "Investment in new AI-based technologies has been one of the critical strategies of the public sector at various levels of government in several countries around the world" [37]. All these examples validate that public agencies are already working with strategic plans. However, the development of CCS in the public sector remains far behind current expectations and opportunities. Due to the provision of resources, public sector agencies still offer most processes in an outdated way [37]. These resources include human capacities such as the recruitment of data scientists, as well as financial resources and the willingness and support of decision-makers to innovate the public sector [36]. Still, as long as CCS are not acknowledged as a major part of digital transformation, public agencies will not receive the necessary attention or resources to be successful.

In summary, while CCSs share some commonalities with other technologies, they are also markedly different. As such, it is necessary to assess them using their own maturity model rather than by using other generic technology maturity models.

\subsection{Levels}

The maturity model levels are discussed below.

\section{Ad-hoc}

Given the interest in CCSs, most public agencies are considering deploying them in some manner. This level is largely driven by hobbyists and those with some personal connection to the problem or technology. Given the early days, it is not surprising to note that some agencies have an ad-hoc approach to CCSs. While these agencies might want to deploy CCSs, they lack the necessary data, technical, personnel and organizational capacities. On the data front, the agencies do not have the necessary data, or access permissions, in place to develop learning algorithms. While efforts might be underway to 
inspect the quality of data, these will be sporadic at best. The technical assets required to develop CCSs are in many cases limited (e.g. trial versions). Access to computational systems is most often restricted to the IT personnel. The agency lacks sufficient analytical capacity and there is no organization-wide strategy in place to address this deficiency. The agency struggles with innovation, especially when it concerns emerging technologies. Another challenge is to balance opportunities due to CCSs affordances and consider the risks in a disciplined manner. There is no systematic processes to promote experimentation with CCSs and as such, most of the current efforts are by guerrilla employees. Formal governance, policy frameworks, or in general ethical frameworks are missing. CCSs are not on the strategic agenda of the agency.

\section{Experimentation}

Agencies that take a deliberate approach to CCSs normally begin at the experimentation level. The big evolution from the prior step is that the organization begins to take notice and preliminary actions on the systems. Agencies commission specific pilot projects to learn about CCSs in a controlled setting. Pilot projects normally focus on either low hangingopportunities or grand challenges. The former become easy testbeds, as agencies can bring its deep knowledge and expertise to bear while the latter, are opportunities for the agency to engage with opportunities by leveraging innovations across their ecosystem. A low hanging opportunity might be the application of robotic process automation to increase effectiveness and efficiency of a manual process. Still, the analytical capacity of the agency will remain low at this level. However, the agency will assign key personnel to pilot projects, which is an important necessary first step. Within the pilot projects, there will be an appetite for innovation, however this will be tempered by the need to deliver quick wins and minimize risk. Ethical frameworks to guide initial experimental accountability are put in place. Leadership of the agency will be kept abreast about pilot projects.

\section{Planning and deployment}

Results from the experimental projects should lead to more detailed planning and deployment of fullfledged CCSs projects. At this stage, the organization is definitely interested in CSSs and has enough experience to begin to understand how to deploy them, their benefits and costs and risks. At this level, public agencies commission one or a few CCSs projects. Datasets required for these projects are assembled. Significant effort is expelled on building these datasets and putting in place appropriate data governance frameworks. If necessary, authorizations to use datasets for CCSs will need to be secured from relevant legal and legislative entities. Significant investments in computational resources will be made at this level. Ideally, the agency should leverage computational resources that are available through strategic partnerships. The agency commits to building up its analytical capacity. Concerted effort is made to increase the analytical capacity through recruitment and public-private partnerships. The innovation climate is favorable when it comes to experiments using CCSs, given the wins secured to date. Agencies formalize innovation processes around CCSs to ensure that they can seek feedback from necessary stakeholders. Opportunities are created to regularly engage internal stakeholders on CCSs. The usage of formal governance and policy frameworks is still sporadic. Accountability of CCSs is formally established. CCSs are now part of strategic planning considerations. While early stage, agency plans now being to account for the potential of CCSs as other strategic considerations (e.g. resourcing) are contemplated. Initial set of metrics are put in place to guide the evaluation of projects and to enable communications.

\section{Scaling and learning}

Agencies that navigate their initial deployments of CSSs successfully should be able to move up to the scaling and learning level. The focus at this level is to extend the scale and scope of CCSs efforts albeit in a disciplined but still cautious manner. Collaboration between the IT department and program/division heads leads to identification and prioritization of opportunities. Data governance protocols are put in place to ensure that CCSs projects launched adhere to necessary legal, ethical, and policy guidelines. Efforts are underway to link datasets across programs, departments, and even with external platforms (e.g. social media platforms). Concerted effort is made to develop guidelines to inspect data for biases and other harms. A systematic effort is undertaken to map out existing computational capabilities and identify areas for technology investments. Programs are put in place to upskill the workforce and develop necessary analytical capacity. Project-level metrics to evaluate CCSs and measure their contribution to advancing public value are designed and evaluated. The usage of formal governance and policy frameworks is mandated on this level. Ethical frameworks are implemented and accountability for CCCs is formally established. There is growing appreciation for the strategic potential of CCSs to transform how the 
agency engages with citizens, delivers services, and designs policy.

\section{Enterprise-wide transformation}

At the enterprise-wide transformation level, the public agency has leveraged lessons learned from its scaling efforts and, at this level, public agencies at this level realize that CCSs can enable organization-wide transformation in how they conduct the business of government. The caution that characterized the prior level is replaced by much more aggressive implementations. Established data governance frameworks enable the agency to extract from, and link, heterogeneous data repositories. Datasets employed by CCSs can be put through the necessary tests to ensure that they meet necessary quality control standards. The agency is following established best practices when it comes to designing computational systems. The agency has agile protocols in place to engage stakeholders (e.g. technology vendors, academia, SMEs, etc.) to access external innovations and capacity as necessary. Computational systems are regularly audited to ensure that they are meeting expected performance standards. The agency has established training programs in place to bolster the analytical capacity of its workforce. A strong innovation climate promotes rapid experimentation with CCSs. Employees see the value in engaging in the design, development, and deployment of CCSs. Ethical frameworks are regularly evaluated and updated. The agency has metrics in place to track, and improve the, performance of CCSs.

\section{Discussion}

This paper presents a maturity model that outlines how the public sector implements CCSs. The model outlines five levels that public agencies move through to fully embedded CCSs in the organizational fabric. Based on our research, we find that public agencies experience different stimuli that contribute to their interest in CCSs and even their move from one level to the next. External pressures (e.g. at the federal level this could be peer agencies adopting CCSs and seeing results or at the local government level it can be efforts at other cities or counties) often act as stimulants for agencies to move from the ad-hoc to the experimentation level. The presence of knowledge sharing networks to facilitate sharing of lessons learned and collaboration with external stakeholders (industry and academia, in particular), are salient for agencies to make the leap from experimentation to planning and deployment. Middle-management who often are put in-charge of pilot projects often remark that their ability to access peers and share results from pilots in a safe environment facilitates learning, adjustments and refinements to pilots, and even gives them the ability to do rough benchmarking.

At the planning and deployment level, it is paramount for agencies to invest the necessary resources to develop thoughtful medium-range plans that outline how efforts on CCSs are aligned to the agency's current and near-term priorities. Doing so, allows the agency to build a solid campaign to keep take stakeholders on the CCSs journey and communicate progress. Collaboration between the IT department and the various program leaders within agency is critical at this stage. Strong business cases that clearly articulate how CCSs advance public value (e.g. streamlined delivery of services, lowering administrative burden) are invaluable here.

Scaling and learning from CCSs requires the agency to have the necessary discipline to invest in learning activities. Often, agencies do not have the necessary resources to reflect on, capture, and even share lessons learned. This results in scaling of ineffective practices, errors, and poor solutions. To ensure that agencies are good stewards of public funds (i.e. lower the expenditure on failed IT projects), a concerted effort is needed on organizational learning. Scaling calls for balancing between exploitation and exploration. As agencies get comfortable with a one class of CCSs, the tendency to simply apply the same tools going forward grows. Agencies must continuously stay abreast of new developments in the CCSs and commission efforts at the experimentation level on these. As such, agencies can use the maturity model to chart how they perform on a set of CCSs and as at the portfolio level of CCSs.

While our model is presented under major categories, this is not be construed as meaning that the elements operate in isolation. Rather, they are richly intertwined and inextricably linked. That is, low organization elements can constrain the technical elements and vice versa. For example, if big data is robust but the organization lacks an innovative culture, the investment in big data is largely wasted as the organization is incapable of leveraging its investment in data. Conversely, if an organization has a strong strategic vision but lacks analytics capability, that visioning is largely wasted. As such, it is probably most accurate to view the organization's maturity as equal to the least mature element of our model.

We need to stress one recurring comment from our professionals: progression from one level of maturity to the next is not a smooth process and generally does not occur in all levels at the same time. However, it is important for those in the public sector to have a maturity model for technology-driven transformations. Our proposed model can help 
managers make good CSS-centric decisions and raise the overall IQ of CCSs deployments in the public sector. By doing so, this may result in less wasting of taxpayer resources due to greater coordination and sharing of lessons learned, improved benchmarking and even more interorganizational collaboration to tackle cross-organizational problems.

\section{Contributions and Limitations}

This research contributes in several aspects. First, this maturity model is the first known attempt to frame the concept of maturity of CSSs in the public sector. While additional work needs to be done to further demonstrate, evaluate and communicate the model, it offers a first attempt to understand the components of maturity from both a technical and organizational framework. Second, it provides a model that is based on both theory and practice, and thus has value in both domains. In the theoretical area, it offers a potential to theories. In the practice arena, it offers a way to begin to measure maturity and to make wise use of resources to improve maturity. Third, it offers a path to move from lower levels of maturity to higher levels. As such, it shines a light on the path to greater maturity, which is likely to lead to better outcomes for citizens.

Like all research, this work has its limitations. First, CCSs development in the public sector is at a very early stage in development and this means that a great deal of work remains to be done to understand maturity. It is likely that, as the domain matures, more iterations of the model will be necessary. Second, while we believe that, our professionals are representative of the public sector; insights from other professionals will provide additional contribution.

The maturity model offers a strong initial attempt to understand how to assess the maturity of CSSs in the public sector. Given the growing importance of CCSs in this sector and their expected costs, benefits and risks, maturity insights may have a significant ability on the performance of the public sector.

\section{References}

[1] http://businessofgovernment.org/report/roadmap-itmodernization-government, accessed 7-12-2020.

[2] Andersen, K.V. and H.Z. Henriksen, "E-government maturity models: Extension of the Layne and Lee model", Government Information Quarterly, 23(2), 2006, pp. 236248.

[3]

https://www.army.mil/article/236583/army_researchers_tak e_proactive_approach_to_cybersecurity, accessed 7-122020.
[4] Arundel, A., C. Bloch, and B. Ferguson, "Advancing innovation in the public sector: Aligning innovation measurement with policy goals", Research Policy, 48(3), 2019, pp. 789-798.

[5] Bason, C., Leading Public Sector Innovation (second Edition): Co-Creating for a Better Society, 2nd edn., Policy Press, Bristol, 2018.

[6] Becker, J., R. Knackstedt, and J. Pöppelbuß, "Developing Maturity Models for IT Management", Business \& Information Systems Engineering, 1(3), 2009, pp. 213-222.

[7] Bedeley, R.T., T. Ghoshal, L.S. Iyer, and J. Bhadury, "Business Analytics and Organizational Value Chains: A Relational Mapping", Journal of Computer Information Systems, 58(2), 2018, pp. 151-161.

[8] Bertot, J.C., U. Gorham, P.T. Jaeger, L.C. Sarin, and H. Choi, "Big data, open government and e-government: Issues, policies and recommendations", Information Polity, 19(1,2), 2014, pp. 5-16.

[9] Bryson, J. and A. Winfield, "Standardizing Ethical Design for Artificial Intelligence and Autonomous Systems", Computer, 50(5), 2017, pp. 116-119.

[10] Bumblauskas, D., H. Nold, P. Bumblauskas, and A. Igou, "Big data analytics: transforming data to action", Business Process Management Journal, 23(3), 2017, pp. 703-720.

[11] Chatfield, A.T. and C.G. Reddick, "Customer agility and responsiveness through big data analytics for public value creation: A case study of Houston 311 on-demand services", Government Information Quarterly, 35(2), 2018, pp. 336-347.

[12] Crosby, P.B., Quality is free: The art of making quality certain, Mentor, New York, 1980.

[13] Czarnitzki, D., P. Hünermund, and N. Moshgbar, "Public Procurement of Innovation: Evidence from a German Legislative Reform", International Journal of Industrial Organization, 71, 2020, p. 102620.

[14] https://hbr.org/2012/10/data-scientist-the-sexiest-jobof-the-21st-century, accessed 7-12-2020.

[15] http://www.businessofgovernment.org/report/deliveringartificial-intelligence-government-challenges-andopportunities, accessed 7-12-2020.

[16] Desouza, K.C. and A. Bhagwatwar, "Citizen Apps to Solve Complex Urban Problems", Journal of Urban Technology, 19(3), 2012, pp. 107-136.

[17] Desouza, K.C., G.S. Dawson, and D. Chenok, "Designing, developing, and deploying artificial intelligence systems: Lessons from and for the public sector", Business Horizons, 63(2), 2020, pp. 205-213.

[18] Dwivedi, Y.K., M. Janssen, E.L. Slade, N.P. Rana, V. Weerakkody, J. Millard, J. Hidders, and D. Snijders, "Driving innovation through big open linked data (BOLD): 
Exploring antecedents using interpretive structural modelling", Information Systems Frontiers, 19(2), 2017, pp. 197-212.

[19] Fatima, S., Kevin C. Desouza, and G.S. Dawson, "How different countries view artificial intelligence", Brookings, 17.6.2020.

[20] Fosso Wamba, S., S. Akter, A. Edwards, G. Chopin, and D. Gnanzou, "How 'big data' can make big impact: Findings from a systematic review and a longitudinal case study", International Journal of Production Economics, 165, 2015, pp. 234-246.

[21] Gajendra, S., B. Xi, and Q. Wang, "E-Government: Public Participation and Ethical Issues", Journal of EGovernance, 35(4), 2012, pp. 195-204.

[22] Gieske, H., M. Duijn, and A. van Buuren, "Ambidextrous practices in public service organizations: innovation and optimization tensions in Dutch water authorities", Public Management Review, 22(3), 2020, pp. 341-363.

[23] Gregor, S. and A. Hevner, "Positioning and Presenting Design Science Research for Maximum Impact", MIS Quarterly, 37(2), 2013, pp. 337-355.

[24] Gupta, S., A.K. Kar, A. Baabdullah, and W.A. AlKhowaiter, "Big data with cognitive computing: A review for the future", International Journal of Information Management, 42, 2018, pp. 78-89.

[25] Humphrey, W.S., Introduction to Software Process Improvement, Defense Technical Information Center, Fort Belvoir, VA, 1993.

[26] Leckel, A., S. Veilleux, and L.P. Dana, "Local Open Innovation: A means for public policy to increase collaboration for innovation in SMEs", Technological Forecasting and Social Change, 153, 2020, p. 119891.

[27] Liptak, A., "Sent to Prison by a Software Program's Secret Algorithms", The New York Times, 1.5.2017.

[28] Lnenicka, M. and J. Komarkova, "Big and open linked data analytics ecosystem: Theoretical background and essential elements", Government Information Quarterly, 36(1), 2019, pp. 129-144.

[29] Mergel, I., "Agile innovation management in government: A research agenda", Government Information Quarterly, 33(3), 2016, pp. 516-523.

[30] Noor, A.K., "Potential of Cognitive Computing and Cognitive Systems", Open Engineering, 5(1), 2014.

[31] Pangburn, D., "San Diego's massive, 7-year experiment with facial recognition technology appears to be a flop", Fast Company, 9.1.2020.

[32] Peffers, K., T. Tuunanen, M.A. Rothenberger, and S. Chatterjee, "A Design Science Research Methodology for Information Systems Research", Journal of Management Information Systems, 24(3), 2007, pp. 45-77.
[33] Piening, E.P., "Dynamic Capabilities in Public Organizations", Public Management Review, 15(2), 2013, pp. 209-245.

[34] Poister, T.H., "The Future of Strategic Planning in the Public Sector: Linking Strategic Management and Performance", Public Administration Review, 70, 2010, s246-s254.

[35] https://www.ibm.com/thought-leadership/institutebusiness-value/report/govchange\#, accessed 7-12-2020.

[36] Sangki, J., "Vision of future e-government via new egovernment maturity model: Based on Korea's egovernment practices", Telecommunications Policy, 42(10), 2018, pp. 860-871.

[37] Sousa, W.G. de, E.R.P. de Melo, P.H.D.S. Bermejo, R.A.S. Farias, and A.O. Gomes, "How and where is artificial intelligence in the public sector going? A literature review and research agenda", Government Information Quarterly, 36(4), 2019, p. 101392.

[38] Sun, T.Q. and R. Medaglia, "Mapping the challenges of Artificial Intelligence in the public sector: Evidence from public healthcare", Government Information Quarterly, 36(2), 2019, pp. 368-383.

[39] van Engelenburg, S., M. Janssen, and B. Klievink, "Design of a software architecture supporting business-togovernment information sharing to improve public safety and security", Journal of Intelligent Information Systems, 52(3), 2019, pp. 595-618.

[40] van Parijs, P., Evolutionary explanation in the social sciences: An emerging paradigm, 1st edn., 1981.

[41] Weerakkody, V., Z. Irani, K. Kapoor, U. Sivarajah, and Y.K. Dwivedi, "Open data and its usability: an empirical view from the Citizen's perspective", Information Systems Frontiers, 19(2), 2017, pp. 285-300.

[42] Zheng, Y., Han, Y., Cui, L., Miao, C., Leung, C., \& Yang, Q., "SmartHS: An AI platform for improving government service provision", The Thirtieth AAAI Conference on Innovative Applications of Artificial Intelligence (IAAI-18), 2018, pp. 7704-7711. 
Table 1. Maturity model for CCSs in the public sector

\begin{tabular}{|c|c|c|c|}
\hline & \multicolumn{3}{|c|}{ Technical Elements } \\
\hline & Big Data & Computational Systems & Analytical Capacity \\
\hline 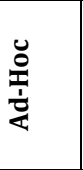 & $\begin{array}{l}\text { - Datasets are extremely limited } \\
\text { - Quality of datasets is unknown and uncertified } \\
\text { - Risks of using datasets incorrectly are high } \\
\text { - Data governance frameworks do not exist }\end{array}$ & $\begin{array}{l}\text { - Required computational systems are not present } \\
\text { - Renegade computational systems are bootstrapped by individual } \\
\text { intrapreneurs } \\
\text { - Computational systems have limited capacity to ingest and } \\
\text { analyse large-scale data }\end{array}$ & $\begin{array}{l}\text { - Agency lacks an organisation-wide view on its analytical capacity or } \\
\text { aptitude. } \\
\text { - Analytical capacity is sparse. } \\
\text { - CCSs developers, data scientists, and other analytical resources learn } \\
\text { by self-teaching or are hobbyist. }\end{array}$ \\
\hline : & $\begin{array}{l}\text { - Datasets are still limited } \\
\text { - Datasets used for experiments go through ad hoc quality control } \\
\text { - Risks of using selected datasets are identified } \\
\text { - Data governance frameworks are constructed around } \\
\text { experimental projects }\end{array}$ & $\begin{array}{l}\text { - Initial prototypes of CCSs are developed and/or acquired but are } \\
\text { still primarily under the radar and are one off systems } \\
\text { - Computational systems have capacity to analyse data in limited } \\
\text { contexts } \\
\text { - CCSs are focused on analysing past data and building } \\
\text { associations between elements of interest (i.e. descriptive } \\
\text { analysis) }\end{array}$ & $\begin{array}{l}\text { - Initial efforts are conducted to assess analytical capacity within the } \\
\text { agency. } \\
\text { - Analytical capacity is centred around pilot projects } \\
\text { - Initial efforts are commissioned for staff to receiving training to } \\
\text { bolster their analytical capacity. }\end{array}$ \\
\hline 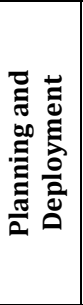 & $\begin{array}{l}\text { - Larger and richer datasets are constructed through fusing data } \\
\text { from heterogeneous systems within the agency. Heavily } \\
\text { structured data. } \\
\text { - All datasets are put through quality control processes but still ad } \\
\text { hoc } \\
\text { - Risk management protocols are designed for selected group of } \\
\text { data assets } \\
\text { - Data governance frameworks around CCSs projects are put in } \\
\text { place }\end{array}$ & $\begin{array}{l}\text { - CCSs are purchased and or licensed by departments and/or teams } \\
\text { within the agency } \\
\text { - Computational systems can ingest and process and large-scale } \\
\text { data } \\
\text { - CCCs are focused not only focused on descriptive analysis but } \\
\text { also on explanatory and predictive insights. }\end{array}$ & $\begin{array}{l}\text { - Agency has an appreciation for its analytical capacity and aptitude } \\
\text { - The agency has the required analytical capacity to undertake an initial } \\
\text { set of operational projects. } \\
\text { - Alliances are formed with external stakeholders to tap into analytical } \\
\text { capacity as needed. } \\
\text { - The agency has identified training resources for staff to bolster their } \\
\text { analytical capacity. }\end{array}$ \\
\hline 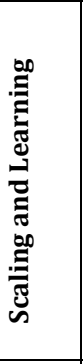 & $\begin{array}{l}\text { - Datasets grow in complexity and also draw from systems, } \\
\text { platforms, and organisations outside the agency. Increasingly } \\
\text { unstructured data. } \\
\text { - Data quality control processes are defined and generally } \\
\text { followed. Processes are in place to learn from their applications } \\
\text { at larger-scales. Risk management protocols are refined to } \\
\text { operate at larger-scales. } \\
\text { - Data governance frameworks are finalized and we begin to see } \\
\text { the emergence of standards that are put in place to ensure } \\
\text { interoperability and seamless integration of data across systems } \\
\text { and environments. }\end{array}$ & $\begin{array}{l}\text { - Emergence of organization wide development and acquisition of } \\
\text { CCSs. A rich ecosystem is developed with external stakeholders } \\
\text { to enable more agile CCSs acquisition and deployment } \\
\text { - The capabilities of CCSs are scaled to take on more data and } \\
\text { different data. } \\
\text { - Predictive analysis and the deployment of autonomous systems } \\
\text { become core foci for CCSs. } \\
\text { - Learning mechanisms are put in place to generate agency-wide } \\
\text { insights form CCSs deployments }\end{array}$ & $\begin{array}{l}\text { - The agency has an organisation-wide view of its analytical capacity } \\
\text { and has a strategy to address the gaps. } \\
\text { - Analytical capacity continues to increase enabling for CCSs to be } \\
\text { deployed in other domains and scaled } \\
\text { - The agency understands how to continue to develop analytical } \\
\text { capacity while balancing its internal investments with the value } \\
\text { provided from alliances with external stakeholders. } \\
\text { - The agency has programs and initiatives to facilitate training and } \\
\text { learning on analytical tools and methodologies. }\end{array}$ \\
\hline 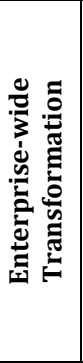 & $\begin{array}{l}\text { - Rich datasets are available that provide insights across } \\
\text { ecosystems of interest. Fully links structured and unstructured } \\
\text { data. } \\
\text { - Data quality standards are established and consistently } \\
\text { followed. Quality control processes are in place and regularly } \\
\text { updated. } \\
\text { - Risk management protocols are in place to oversee data assets } \\
\text { - Data governance frameworks and standards are enterprise-wide } \\
\text { and engrained in the organisational fabric }\end{array}$ & $\begin{array}{l}\text { - Agency-wide policies are in place and followed on CCSs } \\
\text { acquisition, partnerships, and maintenance strategies. A robust } \\
\text { ecosystem of external partners is in place and scanned for } \\
\text { opportunities. } \\
\text { - CCSs have capabilities to ingest emergent data in an agile } \\
\text { manner. } \\
\text { - The entire gamut of analytics and intelligence-driven operations } \\
\text { can be conducted by CCSs } \\
\text { - The agency has and follows a formal process for learning and } \\
\text { improving its practices on CCSs acquisition, deployment, and } \\
\text { maintenance }\end{array}$ & $\begin{array}{l}\text { - The agency constantly monitors its analytical capacity across the } \\
\text { agency and proactively fills gaps as necessary } \\
\text { - Analytical capacity is adequate and distributed across the agency } \\
\text { - The agency has robust alliances with external stakeholders to tap into } \\
\text { analytical capacity in an agile manner. } \\
\text { - Analytical capacity is seen a key asset of the agency and evidence- } \\
\text { driven decision-making permeates the organisation. }\end{array}$ \\
\hline
\end{tabular}




\begin{tabular}{|c|c|c|c|}
\hline & \multicolumn{3}{|c|}{ Organizational Elements } \\
\hline & Innovation Climate & Governance and Ethical Frameworks & Strategic Visioning \\
\hline 峞 & $\begin{array}{l}\text { - No appetite for innovation with CCSs } \\
\text { - Individuals are left to their own to experiment with CCSs } \\
\text { - CCSs are viewed as unacceptably risky } \\
\text { - CCSs deployments are done in the shadows. } \\
\text { - No policies in place to recruit, develop, and retain talent needed to } \\
\text { develop and manage CCSs }\end{array}$ & $\begin{array}{l}\text { - No formal governance and policy frameworks to guide CCSs } \\
\text { - No ethical framework to guide design, development, and deployment } \\
\text { of CCSs } \\
\text { - No accountability for CCSs }\end{array}$ & $\begin{array}{l}\text { - CCSs are not part of the strategic agenda of the agency. } \\
\text { - No funding provided for CCSs efforts. }\end{array}$ \\
\hline 竞 & $\begin{array}{l}\text { - Low innovation appetite but openness to learning about CCSs } \\
\text { - Agency supports innovation on CCSs within controlled settings } \\
\text { - Risk continues to be the most significant factor that dominates } \\
\text { CCSs adoption and use decisions. } \\
\text { - Within pilot projects, focus is on addressing low hanging fruit type } \\
\text { efforts where risk is low and results can be easily demonstrated. } \\
\text { - Initial awareness that plans need to be developed to recruit, } \\
\text { develop, and retain talent needed to develop and manage CCSs }\end{array}$ & $\begin{array}{l}\text { - The need for formal governance and policy frameworks for CCSs is } \\
\text { appreciated and recognized } \\
\text { - High-level and preliminary ethical frameworks to guide initial } \\
\text { experimental projects are put in place } \\
\text { - Formal accountability for CCSs is limited to the teams that are } \\
\text { involved with pilot projects. } \\
\text { - Little formal oversight of governance activities. } \\
\text { - Ethical frameworks are most often derived from other projects } \\
\text { without regard for differences in domains. }\end{array}$ & $\begin{array}{l}\text { - Senior leadership is aware of CCSs pilot projects but are } \\
\text { generally hands-off. } \\
\text { - Limited one-off funding is provided for pilot projects. }\end{array}$ \\
\hline 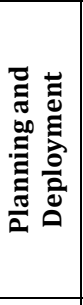 & $\begin{array}{l}\text { - The agency has an appreciation for the value of CCSs and their role } \\
\text { in modernising operations } \\
\text { - Support is provided for innovation in a few targeted areas on CCSs } \\
\text { - Focus shifts from primarily risk-based to cost-based decisions with } \\
\text { CCSs adoption and use decisions. } \\
\text { - Within initial projects and planning, there is a broader appetite for } \\
\text { innovation when it comes to CCSs Initial and limited-scope plans } \\
\text { are developed to recruit, develop, and retain talent needed to } \\
\text { develop and manage CCSs }\end{array}$ & $\begin{array}{l}\text { - Formal governance and policy frameworks to guide CCSs are put in } \\
\text { place but their usage is often sporadic } \\
\text { - Ethical frameworks to guide design, development, and deployment of } \\
\text { CCSs are designed and are starting to be specific to CSSs and not } \\
\text { simply adopted from other domains. } \\
\text { - Accountability for CCSs is formally established and resides within } \\
\text { specific project teams. However, actual accountability is uneven. }\end{array}$ & $\begin{array}{l}\text { - Senior leadership is aware of CCS efforts and are generally } \\
\text { supportive of the initial projects. } \\
\text { - Funding is provided for planning and initial deployments for } \\
\text { CCSs. } \\
\text { - Initial set of metrics to measure CCSs performance and value } \\
\text { are constructed. } \\
\text { - Early mentions of CCSs in key strategy documents appear but } \\
\text { lack sufficient detail. }\end{array}$ \\
\hline 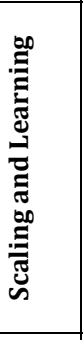 & $\begin{array}{l}\text { - The agency is developing process for surfacing and supporting } \\
\text { innovations on CCSs } \\
\text { - Risk and costs become less of a factor while potential benefits start } \\
\text { to gain prominence in decision-making on CCSs adoption } \\
\text { - The agency has developed collaborative ecosystem to design, } \\
\text { develop, and deploy CCSs with external stakeholders. } \\
\text { - CCSs projects are more ambitious in scope and scale due to the } \\
\text { learnings gained from initial projects. } \\
\text { - Initial plans are scaled to recruit, develop, and retain talent needed } \\
\text { to develop and manage CCSs }\end{array}$ & $\begin{array}{l}\text { - Formal governance and policy frameworks to guide CCSs are in } \\
\text { place and usage is mandated } \\
\text { - Ethical frameworks to guide design, development, and deployment of } \\
\text { CCSs are implemented and generally communicated } \\
\text { - Accountability for CCSs is formally established and resides within } \\
\text { specific departments. } \\
\text { - Accountability principles are in-sync with those in the rest of the } \\
\text { organization. } \\
\text { - Formal training on governance and accountability begins to emerge. }\end{array}$ & $\begin{array}{l}\text { - Senior leadership is aware of and consistently supportive of } \\
\text { CCS project. } \\
\text { - Significant funding is earmarked for long-term CCSs projects. } \\
\text { - Organisational-wide metrics to measure CCSs performance } \\
\text { and value are constricted. } \\
\text { - CCSs are now regular elements of the agency's strategic } \\
\text { planning processes, artefacts (e.g. strategic plans), and } \\
\text { discourse. }\end{array}$ \\
\hline 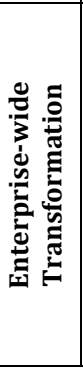 & $\begin{array}{l}\text { - Agency has a fully agreed upon process for surfacing and } \\
\text { supporting innovations in CCSs } \\
\text { - Balanced view of risks, opportunities, costs and benefits in decision } \\
\text { making regarding on CCSs } \\
\text { - The agency continuously monitors its ecosystem for new } \\
\text { opportunities for collaborative alliances to design, develop, and } \\
\text { deploy CCSs with external stakeholders } \\
\text { - CCSs projects span the entire gamut and a balanced portfolio of } \\
\text { CCSs exist across the entire lifecycle. } \\
\text { - Plans are regularly updated to update initiatives and incentives to } \\
\text { recruit, develop, and retain CCSs talent across the agency. }\end{array}$ & $\begin{array}{l}\text { - Formal governance and policy frameworks to guide CCSs are in } \\
\text { place and usage is communicated throughout the organization and are } \\
\text { mandated. } \\
\text { - Ethical frameworks to guide design, development, and deployment of } \\
\text { CCSs are implemented and regularly evaluated and updated. } \\
\text { - Accountability for CCSs is formally established and resides within a } \\
\text { specific department that has the responsibility for agency-wide } \\
\text { coordination and alignment of CCSs activities. } \\
\text { - Widespread training on governance and ethical frameworks is baked } \\
\text { into the organization's training calendar. }\end{array}$ & $\begin{array}{l}\text { - Senior leadership is vocal in their support of CCS projects } \\
\text { - Significant funding is earmarked for long-term CCSs projects. } \\
\text { - Organisational-wide metrics are in place and tracked to } \\
\text { measure CCSs performance and value. Metrics on CCSs } \\
\text { performance and value are regularly communicated to internal } \\
\text { and external stakeholders. } \\
\text { - CCSs are core contributors to achieving an agency's strategic } \\
\text { objectives. }\end{array}$ \\
\hline
\end{tabular}

